13

\title{
Численное исследование убегания электронов в усиленном поле около микроострия
}

\author{
() В.В. Лисенков, ${ }^{1,2}$ С.Н. Иванов, ${ }^{1}$ Ю.И. Мамонтов, ${ }^{2}$ И.Н. Тихонов ${ }^{2}$ \\ ${ }^{1}$ Институт электрофизики УрО РАН, \\ 620016 Екатеринбург, Россия \\ 2 Уральский федеральный университет им. Б.Н. Ельцина, \\ 620002 Екатеринбург, Россия \\ e-mail: lisenkov@iep.uran.ru
}

(Поступило в Редакцию 13 февраля 2018 г.)

Исследован переход автоэмиссионных электронов в режим убегания в области усиленного электрического поля, обусловленного геометрией микроострия на катоде, при различных давлениях газовой среды. Задача решалась моделированием движения электрона в неоднородном электрическом поле методом Монте-Карло в $2 D$-геометрии. В качестве рабочего газа был взят азот. Обнаружено, что прохождение небольшой по размеру области усиленного поля вблизи микроострия может существенно облегчить уход электрона в режим убегания, особенно при давлениях газа свыше $10 \mathrm{~atm}$. По нашему мнению, полученные таким образом убегающие электроны могут создать предварительную ионизацию газовой среды и обеспечить формирование начальной фазы разряда в объемной форме.

DOI: $10.21883 / J T F .2018 .12 .46798 .68-18$

Несмотря на то, что явление убегания электронов в газах высокого давления было обнаружено достаточно давно $[1,2]$, оно является на сегодняшний день одной из наиболее интенсивно исследуемых задач в физике импульсного газового разряда. Уход электрона в режим убегания происходит при условии, когда среднее увеличение энергии электрона в электрическом поле становится больше, чем потери энергии в результате неупругих столкновений с молекулами газовой среды.

Поскольку убегание электронов реализуется при достаточно высоких напряженностях электрического поля [3], в разы превышающих пробивные значения, то генерация убегающих электронов реализуется в разрядах высокого давления, как правило, в отдельных локальных областях разряда, где на короткое время создаются необходимые условия. Поэтому для получения пучков убегающих электронов обычно используются электродные системы с катодом специальной формы, обеспечивающие значительное усиление электрического поля в прикатодной области [2,4-8]. Наиболее полное численное моделирование убегания электронов в подобных системах при высоких давлениях было проведено в работе [9]. Аналитическое исследование выполнено в $[10]$.

Однако в работе [11] в условиях однородного электрического поля во всем промежутке было показано, что убегающие электроны регистрируются при давлениях азота до $4 \mathrm{MPa}$. В работах [12-14] нами было показано, что при давлении $4.5 \cdot 10^{5} \mathrm{MPa} \mathrm{в} \mathrm{случае} \mathrm{плоских} \mathrm{элек-}$ тродов возможна генерация убегающих электронов на завершающей стадии формирования катодного слоя. Но при давлениях $\sim 1 \mathrm{MPa}$ убегания электронов в наших расчетах получено не было.
По нашему мнению подобное расхождение расчетных и экспериментальных результатов объясняется тем, что используемая в расчетах [12-14] одномерная модель оперирует с усредненными характеристиками слоя. При этом все неоднородности на катоде, в частности микроострия, учитываются с помощью усредненного коэффициента усиления, т.е. эмиссия с микроострий однородно распределяется по всей поверхности катода. Подобный подход является не совсем корректным, поскольку не учитывается ускорение электрона в области усиленного поля в окрестности микроострия. Для давлений $\sim 10^{5} \mathrm{~Pa}$ и напряженностей электрического поля в диапазоне $0.01-0.1 \mathrm{MV} / \mathrm{cm}$ это не является критичным, поскольку разность потенциалов, приходящаяся на эту область, в лучшем случае лежит в диапазоне десятков вольт, что практически не прибавляет электрону вероятности в дальнейшем уйти в режим убегания. В этом случае возможность убегания определяется параметрами электрического поля в формирующемся катодном слое. Для давлений $\sim 1 \mathrm{MPa} \mathrm{напряженности} \mathrm{поля} \mathrm{вблизи}$ катода могут достигать $1-10 \mathrm{MV} / \mathrm{cm}$, и электрон, пройдя область усиленного поля в окрестности микроострия, может набрать энергию $100-1000 \mathrm{eV}$, что делает его переход в режим убегания с последующим ускорением вполне вероятным. Для проверки данного предположения нами методом Монте-Карло было проведено моделирование движения электрона, эмиттированного из вершины микроострия в азоте при различных давлениях с целью точного определения условий ухода электрона в режим убегания.

Для моделирования использовались микроострия в форме конуса высотой $h$ и диаметром основания $0.5 h$. Для избежания сингулярностей вершина конуса была скруглена полусферой радиусом $0.01 h$. Наличие такого 


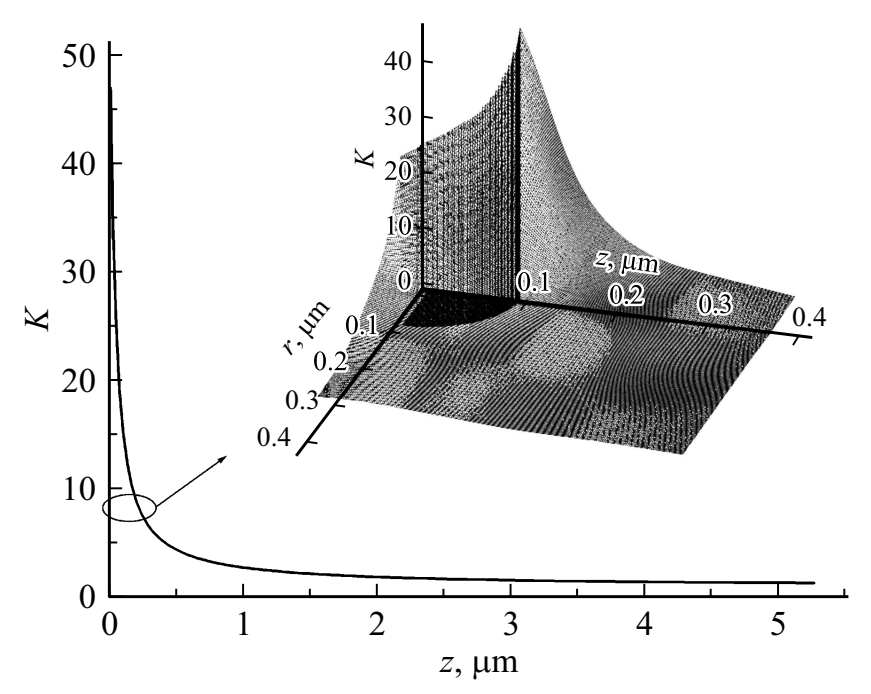

Рис. 1. Характерное распределение коэффициента усиления электрического поля $(K)$ в окрестностях идеально проводящего металлического микроострия высотой $h=10 \mu \mathrm{m}$. На основном графике приведено распределение $K$ вдоль оси конуса $(z)$. За начало координат взята вершина конуса. На вставке приведено пространственное распределение $K$ в области резкого спада вблизи скругленной вершины микроострия. Эта вершина показана в виде темной области с нулевым значением $K$ в начале координат.

микроострия на плоской поверхности катода вызывает искажения электрического поля. Поле вблизи вершины существенно усиливается по сравнению со средним значением поля в межэлектродном промежутке. В таких случаях обычно вводится понятие коэффициента усиления поля $K=E / E_{m}$, где $E$ - локальное значение электрического поля, $E_{m}-$ среднее значение поля в промежутке, равное $E_{m}=U / d$, где $U-$ напряжение на межэлектродном промежутке, $d$ - расстояние катоданод. Для расчета пространственного распределения $K$ использовалось уравнение Лапласа, решаемое в программном пакете ANSYS [15]. Результат такого расчета для $h=10 \mu \mathrm{m}$ приведен на рис. 1 .

Полученное двумерное распределение электрического поля использовалось нами для моделирования движения электрона в пространстве методом Монте-Карло. Для этого использовалась разработанная нами программа, написанная на языке $\mathrm{C}^{++}$, учитывающая набор энергии электрона при его движении между столкновениями и потери в результате неупругих столкновений. При этом характер столкновения (упругое, возбуждение колебательного или электронного уровня, ионизация) разыгрывался с помощью генератора случайных чисел. В качестве газовой среды использовался азот при различных давлениях. Необходимые данные по сечениям были взяты из работ [16-21]. Программа тестировалась для различных значений однородного электрического поля. Полученные константы ионизации и скорости дрейфа электрона неплохо совпали с данными работ $[21,22]$.
На рис. 2 показана зависимость средней напряженности электрического поля (Em) при которой электрон, эмиттированный из вершины микроострия, может перейти в режим убегания. Поскольку такой переход является вероятностным процессом, то за критерий начала перехода мы принимали вероятность больше $1 \%$. Кривым 1 и 2 соответствуют микроострия с высотой $h$ равной $20 \mu \mathrm{m}$ и $10 \mu \mathrm{m}$ соответственно. Для сравнения прямая линия 3 соответствует приведенной напряженности поля начала убегания электронов с той же вероятностью в случае однородного электрического поля (отсутствие микроострий $)$, равной $220 \mathrm{kV} /\left(\mathrm{cm} 10^{5} \mathrm{~Pa}\right)$. Следует заметить, что полученное нами значение несколько меньше полученного также методом Монте-Карло порога убегания в работе [23] $\left(240 \mathrm{kV} /\left(\mathrm{cm} 10^{5} \mathrm{~Pa}\right)\right)$. Это различие, возможно, объясняется разными данными по сечению ионизации. Видно (см. рис. 2), что для давлений менее $0.2 \mathrm{MPa} \mathrm{все} \mathrm{кривые} \mathrm{практически} \mathrm{совпадают.} \mathrm{При}$ более высоких давлениях различия становятся более заметными. В частности, для давления $4 \mathrm{MPa}$ микроострие высотой $20 \mu \mathrm{m}$ дает уменьшение порога убегания в 7 раз, а микроострие с высотой $10 \mu \mathrm{m}$ - в 5 раз по сравнению с однородным полем.

Данный результат объясняется тем, что в случае микроострия на вероятность убегания начинает сказываться не только приведенное, но и абсолютное значение напряженности электрического поля. Электрон, вылетев из микроострия, сразу попадает в область резко усиленного поля, быстро спадающего с расстоянием (см. рис. 1). Эту область он проходит практически без столкновений и приобретает при этом некоторую энергию. Далее он попадает в область меньшего усиления, но более протяженную. Проходя ее, он также ускоряется, но уже с потерями на ионизацию. В итоге после полного про-

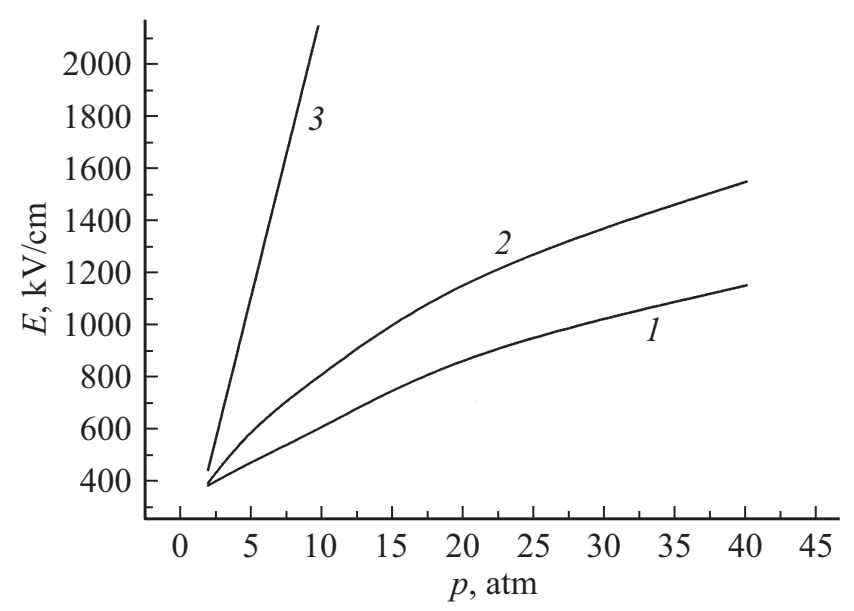

Рис. 2. Зависимость средней напряженности электрического поля $\left(E_{m}\right)$, при которой электроны, эмитированные с поверхности микроострия, начинают уходить в режим убегания от давления $(p)$. Кривая 1 соответствует микроострию высотой $h=20 \mu \mathrm{m}$, кривая $2-h=10 \mu \mathrm{m}$, прямая линия 3 соответствует однородному электрическому полю (отсутствию микроострий). 

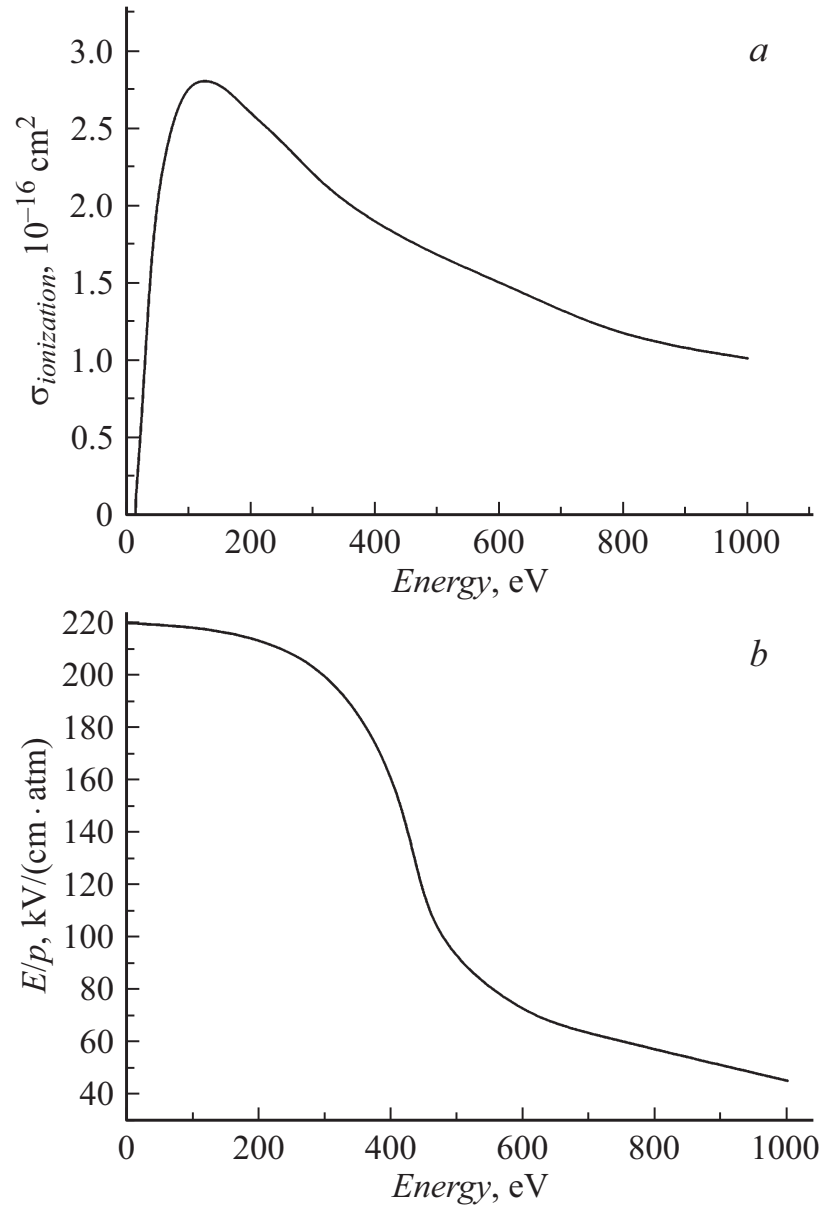

Рис. 3. Сечение ионизации азота в зависимости от энергии $(a)$ [20] и зависимость приведенной напряженности однородного электрического поля, при котором электроны начинают уходить в режим убегания от начальной энергии электрона $(b)$.

хождения области усиленного поля энергия электрона должна превысить энергию максимума сечения ионизации (в случае убегания это основные энергетические потери), т.е. величину $\sim 100 \mathrm{eV}$ (рис. 3,a). Причем, чем больше это превышение, тем больше вероятность ухода электрона в режим убегания в среднем поле разрядного промежутка, т. е. без усиления. Сказанное иллюстрирует рис. $3, b$, на котором показана рассчитанная нами зависимость порогового значения приведенной напряженности электрического поля $(E / p)$ от начальной энергии электрона, при котором становится возможным уход электрона в режим убегания. Видно, например, что при начальной энергии $1000 \mathrm{eV}$ для реализации убегания требуется примерно в 4.5 раза меньшее значение $Q / p$, чем в случае плазменных (или ,медленных“) электронов (электронов с энергиями $\sim 1-10 \mathrm{eV}$ ). С уменьшением начальной энергии электрона пороговые значения $E / p$ возрастают. При энергиях электрона ниже $300 \mathrm{eV}$ пороговые значения $E / p$ практически сравниваются со случаем „медленных“ электронов. Это объясняется тем, что энергетические потери становятся уже достаточно большими и электрон с практически 100\% вероятностью „скатывается“ по энергетической оси в область „медленных“ электронов. Подобный эффект был отмечен ранее в работе [24], однако расчеты там были проделаны для других условий, поскольку решалась иная задача.

Увеличение абсолютного значения напряженности среднего электрического поля в разрядном промежутке и протяженности области локального усиления поля (при увеличении $h$ ) приводит к тому, что электрон после прохождения этой области получает более высокую „начальную“ энергию, что облегчает его дальнейший переход в режим убегания.

Таким образом, высказанное в начале работы предположение о понижении порога убегания при прохождении электроном области усиленного электрического поля, создаваемого микроострием на катоде, качественно подтвердилось последующими расчетами. Единственное несоответствие заключается в том, что эффект становится достаточно заметным при относительно длинных микроостриях (при $h>10 \mu \mathrm{m})$, которые очень редко можно наблюдать на поверхности электродов. Однако нужно учесть то, что электроны, вылетевшие из микроострия и участвуя в лавинном размножении, создают перед ним нескомпенсированный положительный заряд ионов, участвующий в дальнейшем при формировании катодного слоя. Этот заряд приведет к дополнительному усилению поля перед микроострием. Учет этого дополнительного усиления, по нашему мнению, позволит получить убегание в условиях, соответствующих эксперименту [11]. Наличие убегающих электронов на начальной стадии формирования субнаносекундного газового разряда при давлениях выше $1 \mathrm{MPa}$ может способствовать формированию разряда в объемной форме, что позволяет объяснить наблюдаемые в $[13,14,25]$ фоторазвертки свечения разряда и короткие времена запаздывания разряда [26,27], которые невозможно объяснить с помощью лавинно-стриммерной модели. Исследования в этом направлении в настоящее время нами ведутся, и планируется публикация более подробной статьи.

Работа выполнена при поддержке РФФИ (грант № 16-08-00894).

\section{Список литературы}

[1] Станкевич Ю.Л., Калинин В.Г. // ДАН СССР. 1967. Т. 177. C. 72 .

[2] Frankel S., Highland V., Sloan T., Van Dyck O., Wales W. I/ Nucl. Instr. Method. 1966. Vol. 44. P. 345-348.

[3] Бабич Л.П., Лойко Т.В., Цукерман В.А. // УФН.1990. Т. 160. № 7. C. 49-82. [Babich L.P., Loiko T.V., Tsukerman V.A. // Sov. Phys. Usp. 1990. Vol. 33. P. 521-540.]

[4] Месяи Г.А., Коровин С.Д., Шарыпов К.А., Шиак В.Г., Шунайлов С.А., Яландин М.И. // Письма в ЖТФ. 2006. T. 32. Вып. 1. C. 35-44. [Mesyats G.A., Korovin S.D. et al. // Techn. Phys. Lett. 2006. Vol. 32. P. 18-22.] 
[5] Месяи, Г.А., Шиак В.Г., Шунайлов С.А., Яландин М.И. // Письма в ЖТФ. 2008. Т. 34. Вып. 4. С. 71-80. [Mesyats G.A., Shpak V.G. et al. // Techn. Phys. Lett. 2008. Vol. 34. P. 169173.]

[6] Костыря И.Д., Бакшт Е.Х., Тарасенко В.Ф. // ПТЭ. 2010. № 4. C. 84-87. [Kostyrya I.D., Baksht E.Kh., Tarasenko V.F. // Instrum. Exp. Techn. 2010. Vol. 53. P. 545548.]

[7] Тарасенко В.Ф. // Физика плазмы. 2011. Т. 37. № 5. С. $444-$ 457. [Tarasenko V.F. // Plasma Physics Reports. 2011. Vol. 37. P. 409-421.]

[8] Mesyats G.A., Sadykova A.G. et al. // IEEE Trans. on Plasma Sci. 2013. Vol. 41. N 10. Pt 1. P. 2863.

[9] Shklyaev V.A., Belomyttsev S.Ya., Ryzhov V.V. // J. Appl. Phys. 2012. Vol. 112. P. 113303.

[10] Зубарев Н.М., Иванов С.Н. // Физика плазмы. 2018. Т. 44. № 4. C. 397-406. [Zubarev N.M., Ivanov S.N. // Plasma Physics Reports. 2018. Vol. 44. P. 445-452.]

[11] Ivanov S.N. // J. Phys. D: Appl. Phys. 2013. Vol. 46. P. 285201.

[12] Иванов С.Н., Лисенков В.В., Шиак В.Г. // ЖТФ. 2008. Т. 78. Вып. 9. C. 62-68. [Ivanov S.N., Lisenkov V.V., Shpak V.G. // Tech. Phys. 2008. Vol. 53. N 9. P. 1162-1168.]

[13] Иванов С.Н., Лисенков В.В. // ЖТФ. 2010. Т. 80. Вып. 1. C. 54-58. [Ivanov S.N., Lisenkov V.V. // Tech. Phys. 2010. Vol. 55. N 1. P. 53-57.]

[14] Ivanov S.N., Lisenkov V.V., Shpak V.G. // J. Phys. D: Appl. Phys. 2010. Vol. 43. P. 315204.

[15] Огородникова О.М. // Информационные технологии в проектировании и производстве. 2014. № 2. С. 3034. [Ogorodnikova O.M. // Information Technology of CAD/CAM/CAE. 2014. N 2. P. 30-34.]

[16] Engelhardt A.G., Phelps A.V., Risk C.G. // Phys. Rev. 1964. Vol. 135. P. A1566-1574.

[17] Schulz G.J. // Phys. Rev. 1964. Vol. 135. P. A988-994.

[18] Cartwright D.C. // Phys. Rev. A. 1977. Vol. 16. P. 1013-1040.

[19] Cartwright D.C. // Phys. Rev. A. 1977. Vol. 16. P. 1041-1051.

[20] Kieffer L.J., Dunn G.H. // Rev. Mod. Phys. 1966. Vol. 38. P. 1-35.

[21] Phelps A.V., Pitchford L.C. // Phys. Rev. A. 1985. Vol. 31. P. 2932-2949.

[22] Jelenkovic B.M., Phelps A.V. // Phys. Rev. A. 1987. Vol. 36. P. 5310-5326.

[23] Bakhov K.I., Babich L.P., Kutsyk I.M. // IEEE Trans. on Plasma Sci. 2000. Vol. 28. P. 1254-1262.

[24] Ткачев А.Н., Яковленко С.И. // Письма в ЖТФ. 2006. Т. 32. Вып. 13. C. 37-42. [Tkachev A.N., Yakovlenko S.I. // Tech. Phys. Lett. 2006. Vol. 32. P. 572.]

[25] Иванов С.Н. // ДАН. 2004. Т. 399. № 4. С. 472-476. [Ivanov S.N. // Dokl. Phys. 2004. Vol. 49. P. 701-705.]

[26] Иванов С.Н., Шарыпов К.А. // Письма в ЖТФ. 2016. Т. 42. Вып. 5. C. 102-110. [Ivanov S.N., Sharypov K.A. // Tech. Phys. Lett. 2016. Vol. 42. P. 274-277.]

[27] Иванов С.Н., Лисенков В.В. // Физика плазмы. 2018. Т. 44. № 3. C. 1-10. [Ivanov S.N., Lisenkov V.V. // Plasma Phys. Rep. 2018. Vol. 44. N 3. P. 1-10.] 